\title{
LEVOSIMENDAN EN DISFUNCIÓN VENTRICULAR EN EL POSOPERATORIO DE CIRUGÍA CARDIACA HOSPITAL SANTA CLARA DE BOGOTÁ DC. OCTUBRE 2011 A SEPTIEMBRE 2012
}

Expedito Badillo MD*, José María Hennessey MD**, Edward Alonso Santis MD**

\section{Resumen}

La disfunción ventricular después de circulación extracorpórea causa síndrome de bajo gasto cardiaco en el posoperatorio (prevalencia de $\mathbf{1 0 \%}$ ). Levosimendan es un medicamento para mejorar la disfunción ventricular refractaria. Objetivo: describir la evolución de la función ventricular en adultos después de cirugía cardiaca manejados con levosimendan por disfunción ventricular refractaria en el Hospital de Santa Clara de Bogotá DC, entre octubre 2011 y septiembre de 2012. Métodos: se reportan las características clínicas y la evolución ecocardiográfica de la fracción de eyección (FEVI) con control a las 24 horas y a la semana del posoperatorio. Resultados: se evaluaron 13 pacientes con edad media de 59 años (DE; 10), rango de 39 a 78 años, nueve fueron hombres, dos presentaban diabetes mellitus. La fracción mediana de eyección ventricular izquierda prequirúrgica fue de 50\% (RIQ 30\%-60\%). Como respuesta a la administración de levosimendan la mediana de la FEVI a las 24 horas fue $20 \%$ (RIQ $20 \%-25 \%$ ) y $35 \%$ a la semana (RIQ $35 \%$ - $40 \%$ ). Dos pacientes fallecieron. Conclusiones: la utilización del levosimendan en pacientes con disfunción ventricular izquierda refractaria contribuye a la mejoría de la fracción de eyección.

Palabras clave: levosimendan, disfunción ventricular, fracción de eyección.

\section{USE OF LEVOSIMENDAN AFTER CARDIAC SURGERY IN PATIENTS WITH IMPAIRED VENTRICULAR FUNCTION AT HOSPITAL SANTA CLARA, BOGOTÁ DC, COLOMBIA}

\section{Abstract}

\begin{abstract}
Impaired ventricular function after extracorporeal circulation causes postoperative low cardiac output syndrome (prevalence 10\%). Levosimendan is an agent administered to improve refractory impaired ventricular function. $O b$ jective: To describe progress of ventricular function in adult cardiac surgery patients managed with levosimendan for refractory impaired ventricular function, at Hospital de Santa Clara Bogotá DC, between October 2011 and September 2012. Methods: Assessment of clinical features and echocardiographic progress of the ejection fraction (LVEF) 24 hours and one week after surgery. Results: Thirteen (13) patients, mean age 59 years (SD; 10), ranging between 39 to 78 years were included, nine were men, two with diabetes mellitus. The median left ventricle ejection fraction before surgery was 50\% (QRI 30\%-60\%). Following levosimendan administration the median LVEF 24 hours after surgery was $20 \%$ (QRI 20\%-25\%) and 35\% (QRI 35\% -40\%) one week after surgery. Two patients died. Conclusions: The administration of levosimendan in patients with refractory impaired left ventricular function contributes to improve the ejection fraction.
\end{abstract}

Key words: Levosimendan, impaired ventricular function, ejection fraction

Fecha recibido: enero 16 de 2015 - Fecha aceptado: marzo 20 de 2015

* Exjefe de Cirugía Cardiovascular, Hospital de San José. Cirujano cardiovascular, Hospital Santa Clara. Bogotá DC, Colombia.
** Cirujano cardiovascular, Fundación Universitaria de Ciencias de la Salud. Bogota DC, Colombia. 


\section{Introducción}

En Estados Unidos se calcula que $20 \%$ de la población para 2020 tendrá más de 65 años y 5.5\% será mayor de 80 años; se estima para 2050 que los mayores de 80 años serán 25 millones de personas de los cuales el $40 \%$ tendrá enfermedad cardiovascular grave, siendo la causa de muerte más frecuente en este grupo etario. ${ }^{1}$ En Colombia el DANE establece que para 2020 el promedio de vida será 76 años. ${ }^{2}$

Los pacientes intervenidos por cirugía cardiaca bajo circulación extracorpórea que presentan isquemia miocárdica global por pinzamiento aórtico, muestran grados variables de disfunción ventricular refractaria en el posoperatorio inmediato en ausencia de infarto de miocardio. ${ }^{1,3}$ Esta es transitoria pero puede provocar un síndrome de bajo gasto en el posoperatorio. La prevalencia del síndrome es de $10 \%$ en los pacientes intervenidos y la tasa de mortalidad llega a $17 \% .^{4} \mathrm{La}$ isquemia-reperfusión ocasionada por el pinzamiento aórtico genera una disfunción ventricular refractaria debida a la depleción de fosfatos de alta energía, sobrecarga intracelular de calcio, generación de radicales libres y alteraciones de la microcirculación coronaria. ${ }^{5}$ Las fibras musculares lisas de la pared vascular ocasionan estas acciones por un efecto vasodilatador sistémico y coronario ${ }^{6,7}$ e intervienen en el preacondicionamiento isquémico, el aturdimiento miocárdico, en la lesión por isquemia-reperfusión y la apoptosis de los miocardiocitos. ${ }^{8}$ A pesar de los avances en las técnicas quirúrgicas y en los métodos para protección miocárdica, la disfunción ventricular izquierda refractaria sigue siendo un problema común en el posoperatorio de cirugía cardiaca. ${ }^{9}$ Tanto el miocardio aturdido como el hibernante representan tejido viable. ${ }^{10}$ La disfunción miocárdica posoperatoria puede ser similar al "miocardio aturdido", que se define como disfunción miocárdica posisquémica, sistólica o diastólica, que no se asocia con cambios morfológicos (necrosis) y es reversible tras un periodo de convalecencia. Después de cirugía cardiaca la disfunción posisquémica puede ser pasajera cuando en el preoperatorio el corazón era normal $^{11,12}$, pero puede ser una complicación grave en aquellos con disfunción ventricular izquierda preexistente. ${ }^{12}$ El corazón contundido poscirugía cardiaca se acompaña tanto de la disfunción regional ${ }^{11,12}$ como global, ya que las degeneraciones en el movimiento local de la pared pueden impactar en la función global. ${ }^{13,14} \mathrm{El}$ fenómeno de la disfunción poscardioplejia o "corazón aturdido" se puede observar en un amplio espectro de condiciones quirúrgicas, en las que el paro cardiopléjico y la isquemia se usan de rutina como en la cirugía de revascularización coronaria, cambios valvulares y trasplante cardiaco. ${ }^{15}$

\section{Causas de disfunción refractaria posoperatoria}

La cirugía cardiaca por sí sola puede causar daño miocárdico, como las alteraciones en la geometría ventricular por la colocación de prótesis rígidas en el corazón en las intervenciones de cambio valvular. ${ }^{16,17}$ durante la circulación extracorpórea cuando el corazón está sometido a un número de eventos que pueden llevar a una isquemia del miocardio por perfusión miocárdica inadecuada, distensión o colapso ventricular, embolia coronaria, fibrilación ventricular así como el clampeo de la aorta y la reperfusión. Además, la circulación extracorpórea por si sola puede causar disfunción del miocardio como resultado de una hemodilución severa o una hipotermia e incluso una hipercalemia sistemática inducida por las soluciones de cardioplejia. La operación original diseñada para preservar o mejorar la función del miocardio puede estar asociada con efectos deletéreos. El daño por isquemia-reperfusión se produce también durante la infusión intermitente de solución cardiopléjica o por una mala distribución de la misma a todas las áreas del miocardio, mecanismos que contribuyen a la disfunción poscardiopléjica. La administración en grandes dosis puede ocasionar hipocalcemia o hípercalemia sistémica y agravar la disfunción ventricular. ${ }^{16,18,19}$ El pinzamiento aórtico produce modificaciones en el ventrículo izquierdo durante la cirugía cardiaca al causar una disminución en la distensibilidad; sin embargo, se ha observado que estos cambios revierten en las primeras horas, a menos que el pinzamiento se prolongue más de 35 minutos. La hipotermia en la circulación extracorpórea disminuye la tasa metabólica y los requerimientos de oxígeno, por lo tanto reduce la tolerancia a la isquemia lo cual lleva a una depresión 
miocárdica mayor y a trombocitopenia. ${ }^{19}$ Las lesiones por reperfusión se definen como el daño miocárdico que ocurre durante la restauración del flujo sanguíneo al músculo isquémico. La formación de radicales libres de oxígeno de una lipoperoxigenación terminan en una disfunción miocárdica. La lesión por reperfusión tiene una fase temprana (menos de 4 horas) y otra tardía (4 a 6 horas). Incluyen un deterioro estructural (edema, depósito de plaquetas) y una fase bioquímica (disminución en la utilización de oxígeno, activación del complemento, acidosis) y una patología electromecánica (arritmias, disfunción sistólica /diastólica). ${ }^{20}$

Es importante reconocer el síndrome de bajo gasto, debido a la fuerte correlación entre el índice cardiaco en el posoperatorio temprano y la probabilidad de muerte después de la cirugía cardiaca. Por lo regular este síndrome incluye extremidades frías, piel marmórea, presión sistólica baja ( $<90 \mathrm{~mm} \mathrm{Hg}$ ), disminución del gasto urinario ( $<30 \mathrm{ml} / \mathrm{hora})$, bajo índice cardiaco $\left(<2 \mathrm{~L} / \mathrm{min} / \mathrm{m}^{2}\right)$, baja saturación de oxígeno en sangre venosa $(<50 \%)$ y acidosis metabólica. Ante este evento se deben evaluar los parámetros hemodinámicos e integrar la información con hallazgos ecocardiográficos a la cabecera del paciente para confirmar el síndrome de bajo gasto y así establecer un patrón hemodinámico (baja precarga, cardiogénico o séptico). ${ }^{21}$

\section{Farmacodinamia del levosimendan}

Es un calcio sensibilizante de los miofilamentos cardíacos que produce un aumento de la contractilidad miocárdica, con una acción adicional en los canales de potasio sensibles a ATP en el músculo liso vascular que le confiere, además, efectos vasodilatadores. A este tipo de fármacos con acción dual inotrópica positiva y vasodilatadora se les conoce como inodilatadores. ${ }^{20}$

La farmacodinamia del levosimendan potencia la sensibilidad al calcio de las proteínas contráctiles mediante la unión a la tropina $\mathrm{C}$ cardiaca, por un mecanismo dependiente del calcio. Aumenta la fuerza de contracción, pero sin afectar la relajación ventricular. Además, abre los canales de potasio sensibles al ATP en el músculo liso vascular, lo que propicia la dilatación de los vasos arteriales de resistencia sistémicos y coronarios, así como los vasos venosos sistémicos de capacitancia. En pacientes con disfunción ventricular las acciones inotrópicas positivas y vasodilatadoras de levosimendán producen un aumento de la fuerza contráctil y una reducción tanto de la precarga como de la poscarga, sin afectar en forma negativa la función diastólica. ${ }^{22}$ La farmacocinética del levosimendán es lineal en el rango de dosis terapéutica de 0.05-20 microgramos por kilogramo por minuto: su volumen de distribución aproximado (Vss) es de 0.2 litros por kilogramo; se une 97 a $98 \%$ a las proteínas plasmáticas, en especial a la albúmina; la unión de su metabolito activo (OR-1896) es $40 \%$. El fármaco se metaboliza por completo y solo cantidades insignificantes se eliminan en la orina o heces sin modificar, en especial mediante conjugados cíclicos o $\mathrm{N}$-acetilado cisteinilglicina y cisteína. El levosimendan ha sido evaluado en estudios clínicos que han involucrado más de 2.800 pacientes con insuficiencia cardiaca. La eficacia y seguridad cuando esta cursa con descompensación produce aumento de la contractilidad miocárdica con una acción adicional en los canales de potasio sensibles a ATP en el músculo liso vascular que le confiere efectos vasodilatadores. Las dosis de carga intravenosa ( 3 a $24 \mathrm{mg} / \mathrm{k}$ ) o por infusión continua $(0.05$ a $0.2 \mathrm{mg} / \mathrm{k} / \mathrm{min})$ evidencian en estudios importantes su eficacia en el manejo de la disfunción ventricular cuando otros medicamentos no lo han sido. ${ }^{22,23,24}$

Dentro de un rango de dosificación recomendado el levosimendan genera un metabolito terapéuticamente activo cuyo efecto se prolonga entre 7 y 9 días luego de descontinuar la infusión realizada durante 24 horas. El medicamento incrementa el flujo sanguíneo coronario en pacientes que se están recuperando de cirugía coronaria y mejora la perfusión en insuficiencia cardiaca. Estos beneficios se logran sin un aumento significativo en el consumo de oxígeno por el miocardio. Además, la infusión de levosimendan reduce en forma significativa los niveles circulantes de endotelina- 1 en casos de insuficiencia cardiaca congestiva y a las tasas de infusión recomendadas no aumentan los niveles plasmáticos de catecolaminas. Su uso en acontecimientos agudos posoperatorios de cirugía cardiaca no ha sido bien valorado. ${ }^{23,24}$ 
El objetivo de esta investigación es describir la evolución de la función ventricular cardiaca en pacientes adultos a quienes se les realizó cirugía cardiaca y fueron manejados con levosimendan por disfunción ventricular refractaria en el Hospital de Santa Clara de Bogotá DC entre octubre 2011 y septiembre 2012.

\section{Métodos}

Se describe una serie de casos en la que se incluyeron pacientes adultos sometidos a cirugía cardiaca con circulación extracorpórea en el Hospital Santa Clara de Bogotá DC, Colombia, que presentaron disfunción ventricular refractaria definida como disfunción miocárdica posisquémica, sistólica o diastólica, que no se asoció con cambios morfológicos (necrosis) y fue reversible después de un periodo de convalecencia. Los pacientes se manejaron con levosimendan en dosis estándar, describiendo la evolución de la función ventricular cardiaca durante la primera semana. Se excluyeron los que recibieron el fármaco en el preoperatorio, aquellos con choque cardiogénico y a quienes se les implantaron dispositivos de asistencia ventricular o balón de contrapulsación en el pre o transoperatorio.

Para la recolección de datos se implementó un formato de revisión de historias clínicas que incluyó variables demográficas, comorbilidades, procedimientos, tiempos de bomba y de clampeo, euroscore, fracción de eyección del ventrículo izquierdo preoperatorio, FEVI a las 24 horas y a la semana posoperatoria, ritmo cardiaco a la salida de circulación extracorpórea, fibrilación auricular trans y posoperatoria, presencia de infarto de miocardio trans y posoperatorio, índice cardiaco, gasto cardiaco y gasto urinario antes de la administración del levosimendan y 24 horas después. Se reporta la mortalidad intrahospitalaria y los resultados se resumen en frecuencias absolutas y relativas, medidas de tendencia central y de dispersión. El análisis estadístico se efectuó mediante el programa estadístico stata 12. El protocolo fue aprobado por el comité de ética y de investigación del Hospital Santa Clara y el de investigaciones de la Facultad de Medicina de la Fundación Universitaria de Ciencias de la Salud.

\section{Resultados}

La población total fue de 13 pacientes con promedio de edad de 59 años (DE: 10) (39 a 78 años), nueve de género masculino y todos pertenecían al estrato socioeconómico 2 . Siete pacientes presentaban clase función NYHA II, cuatro NYHA III, dos clases función NYHA IV y las comorbilidades identificadas fueron diabetes mellitus en dos casos e hipotiroidismo en uno. Solo dos tenían antecedente de cirugía cardiaca previa de tipo valvular, todos cursaban con una mediana del euroscore de 8\% (RIQ: 3\%-12\%), en seis de los cuales era menor de $5 \%$, tres entre 6 y $10 \%$ en cuatro fue mayor de $10 \%$.

De los procedimientos realizados la revascularización miocárdica corresponde a seis cirugías, reemplazo valvular cuatro y remplazo valvular con revascularización miocárdica tres. Se remplazaron tres válvulas aórticas, tres mitrales y una tricuspídea, utilizando dos válvulas biológicas y cinco mecánicas. La mediana del tiempo de clampeo en la revascularización miocárdica en los seis pacientes fue de 61 minutos con una mínima de 18 y máxima de 76, la CEC presentó una mediana de 91 minutos con una mínima de 25 y máxima de 126, en el remplazo valvular la mediana del tiempo de clampeo fue de 117 minutos con una mínima de 68 y una máxima de 156, la mediana del tiempo de CEC fue de 143 con una mínima de 104 minutos y una máxima de 223, en la revascularización miocárdica con remplazo valvular la mediana del tiempo de clampeo fue de 114 minutos con una mínima de 93 y una máxima de 164, la mediana del tiempo de CEC fue de 180 con una mínima de 151 y una máxima de 198 (Figuras 1 y 2).

Antes de la cirugía cinco pacientes cursaban con trastornos del ritmo, durante la cirugía seis desarrollaron fibrilación auricular y en cinco ocurrió en el posoperatorio. El infarto agudo de miocardio transoperatorio se presentó en un paciente y en dos casos en el posoperatorio.

Los pacientes que recibieron levosimendan y desarrollaron disfunción ventricular izquierda posoperatoria tenían una mediana de fracción de eyección ventricular izquierda prequirúrgica de $46 \%$, seis de ellos presenta 


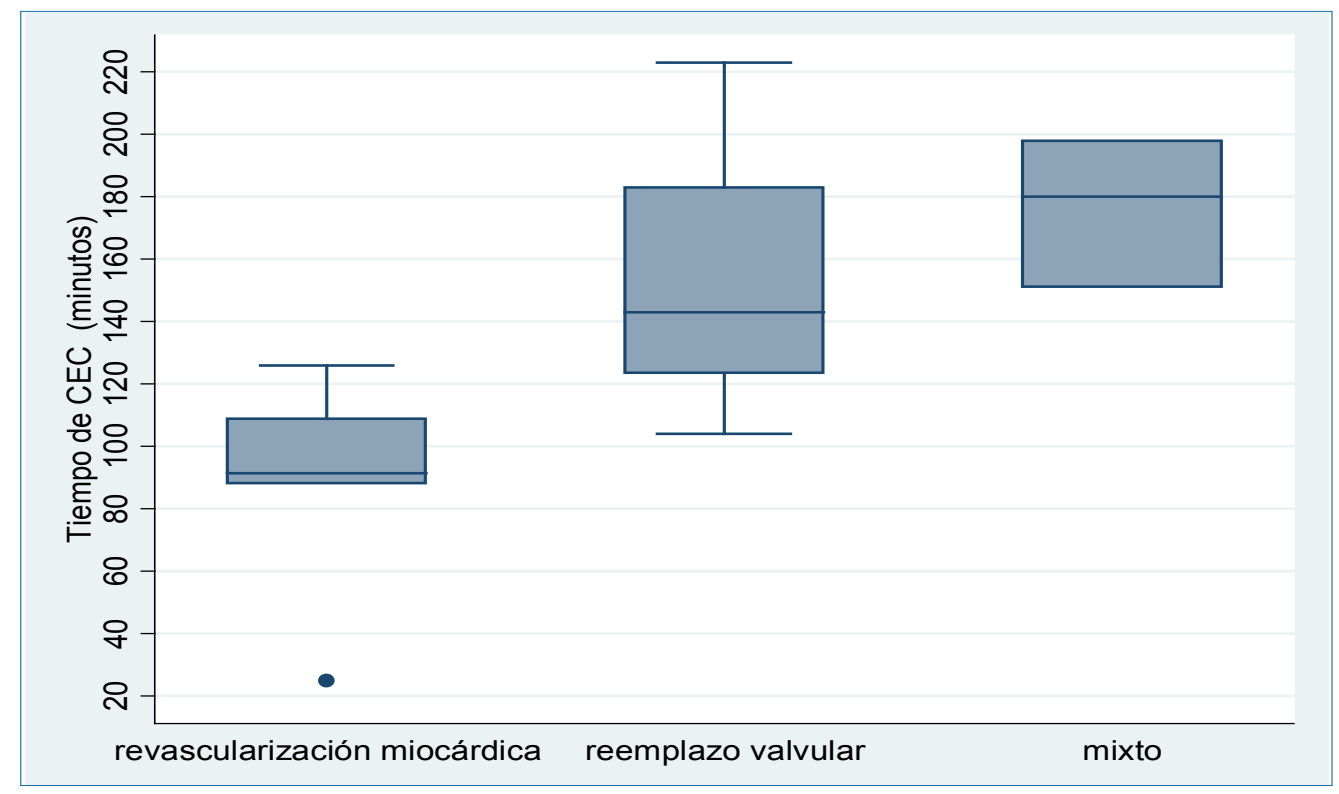

Figura I. Tiempo de bomba según procedimiento.

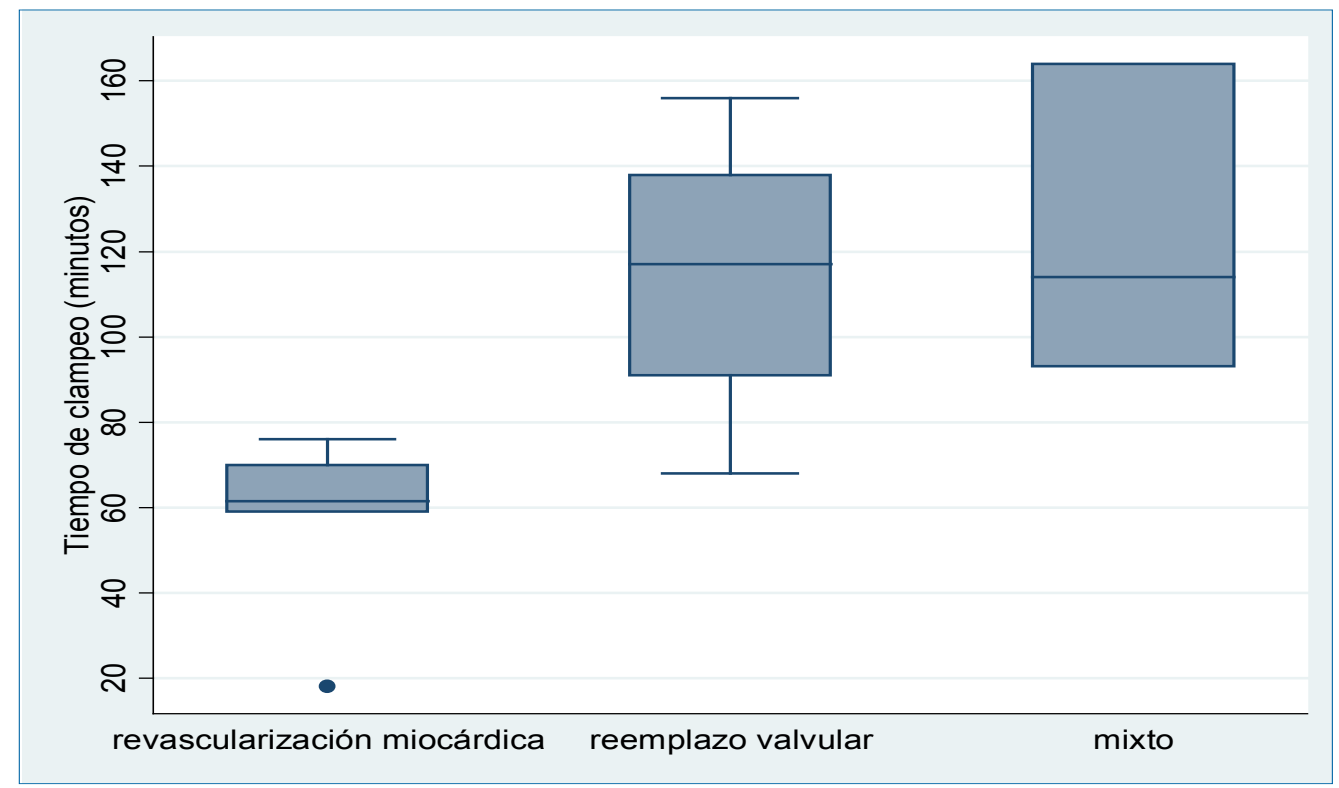

Figura 2. Tiempo de clampeo según procedimiento.

entre $30 \%$ y $40 \%$ y en siete fue mayor de $40 \%$. Los resultados de la FEVI en las primeras 24 horas después de recibir el medicamento revelaron fracción de eyección ventricular mediana de 25\% (RIQ: 20-30\%), de los cuales siete entre 20 y $24 \%$, cinco entre 25 y $29 \%$ y solo uno tenía $30 \%$. En el control ecocardiográfico a la semana de seguimiento se observó una mediana de fracción de eyección ventricular izquierda de $35 \%$ (RIQ:35\%-40\%), dos con valores entre 31 y $34 \%$, seis entre 35 y $39 \%$, dos entre 40 y $44 \%$ y en solo un paciente era mayor de $45 \%$. Se evidenció mejoría de la fracción de eyección ventricular izquierda de $10 \%$ en dos pacientes, $15 \%$ en seis $20 \%$ en dos y solo uno le optimizó un $25 \%$.

La mediana del índice cardiaco en los pacientes antes de recibir el medicamento fue de $2,7 \mathrm{~L} / \mathrm{min} / \mathrm{m}^{2}$ (RIQ: 2.5- $3 \mathrm{~L} / \mathrm{min} / \mathrm{m}^{2}$ ), el gasto cardiaco mostró una media- 
na de $3.5 \mathrm{~L} / \mathrm{min}$ (RIQ: 3 - 3.7 L/min) y el gasto urinario una de $0.7 \mathrm{~mL} / \mathrm{k} /$ hora (RIQ: 0.5 - $0.8 \mathrm{~mL} / \mathrm{k} / \mathrm{hora}$ ).

El tiempo mediano de administración de levosimendan fue 48 horas (RIQ: 24 - 48 horas), siguiendo un control hemodinámico estricto, observando a las 24 horas el índice cardiaco con una mediana de $3.5 \mathrm{~L} /$ $\mathrm{min} / \mathrm{m}^{2}$ (RIQ:3.3 - $3.8 \mathrm{~L} / \mathrm{min} / \mathrm{m}^{2}$ ), el gasto cardiaco una de $5 \mathrm{~L} / \mathrm{min}$ (RIQ: 4.5 - $5 \mathrm{~L} / \mathrm{min}$ y el gasto urinario una de $1 \mathrm{ml} / \mathrm{k} /$ hora (RIQ: $0.9-1.5 \mathrm{~mL} / \mathrm{k} / \mathrm{hora}$ ) (Figuras 3,4 y 5).

Fallecieron dos pacientes en el posoperatorio inmediato, ambos del sexo femenino, una joven y otra añosa, con euroscore alto $(>11)$ llevadas a reintervención por disfunción valvular protésica.

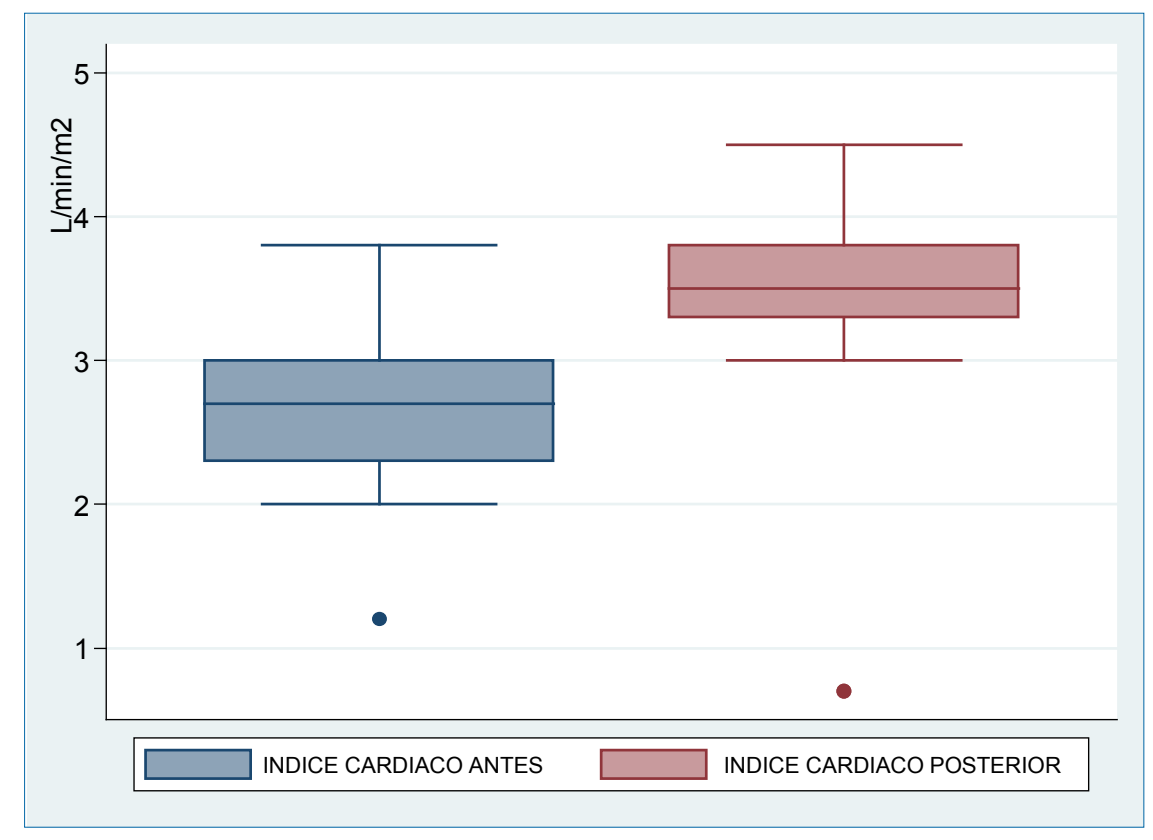

Figura 3. Índice cardiaco antes y después de la administración de levosimendan.

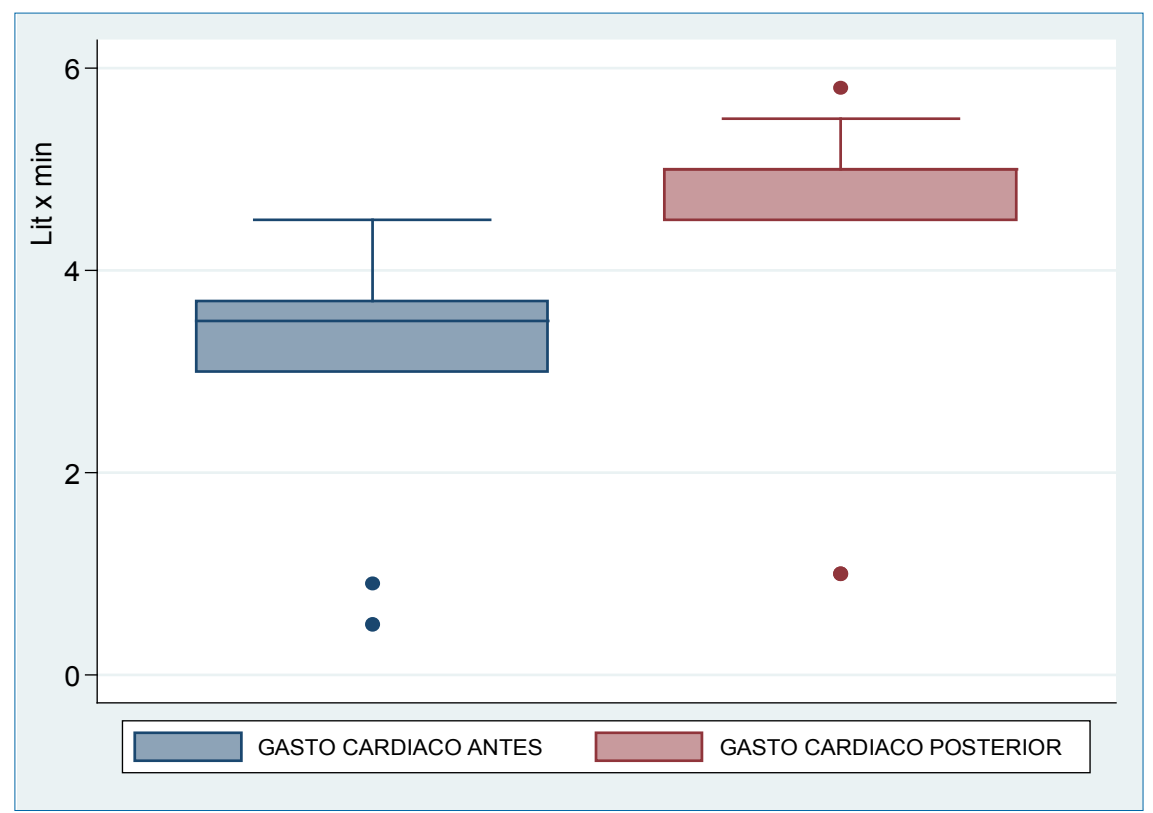

Figura 4. Gasto cardiaco antes y posterior a la administracion de levosimendan. 


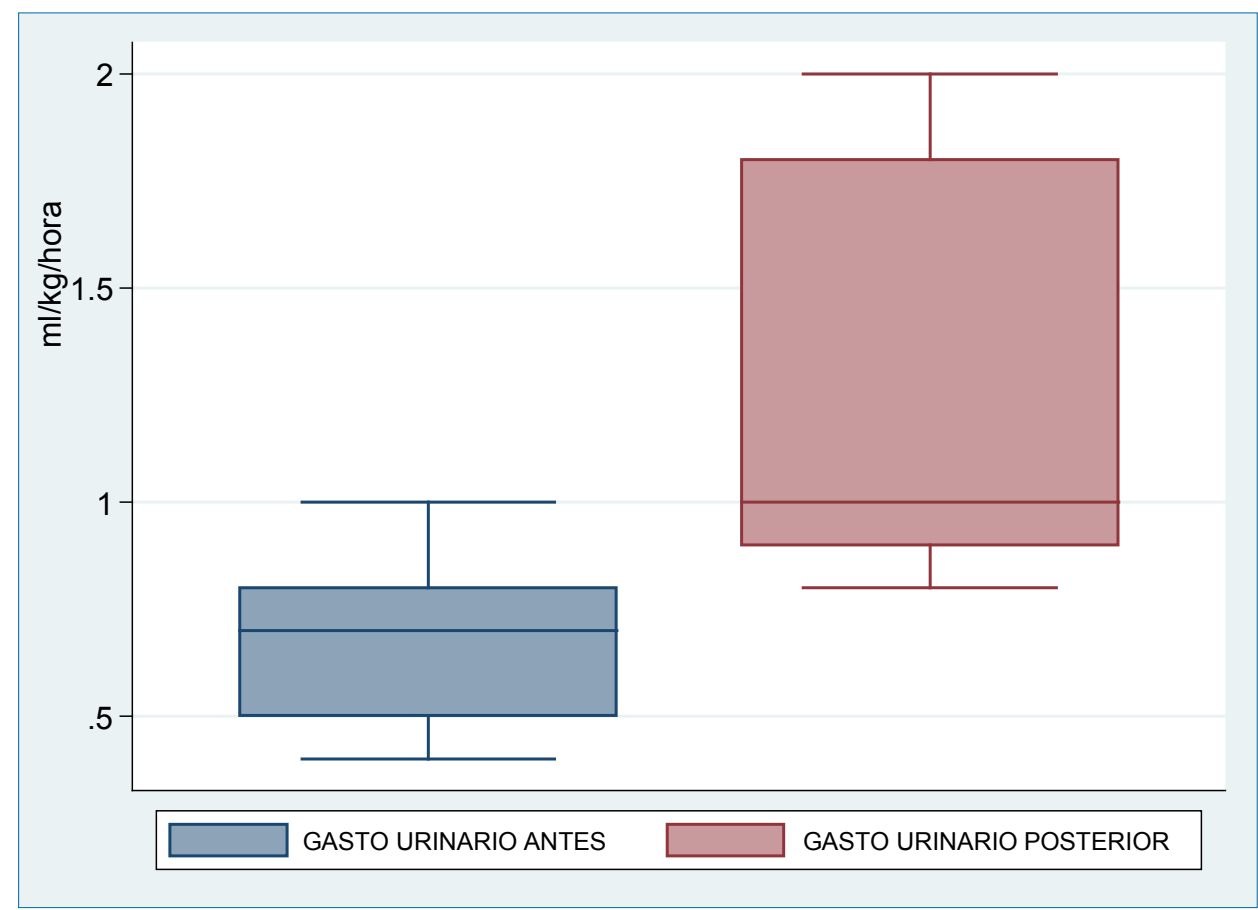

Figura 5. Gasto urinario antes y posterior a la administracion de levosimendan.

\section{Discusión}

Los pacientes que presentaron disfunción ventricular refractaria en el posoperatorio de cirugía cardiaca y que cursaron con síndrome de bajo gasto tenían tratamiento avanzado establecido en la infusión de agentes inotrópicos positivos como beta agonistas, digital o inhibidores de la fosfodiesterasa III. Cualquiera de estas familias de fármacos incrementa el consumo de energía celular y del calcio intracelular aumentando la posibilidad de arritmias. ${ }^{24} \mathrm{El}$ levosimendan está dentro de la familia de los vasodilatadores e inotrópicos que actúa a través de un mecanismo central o de regulación descendente. ${ }^{25}$ En dosis terapéuticas potencia la contractilidad miocárdica sin aumentar la demanda de oxígeno, produce vasodilatación coronaria y sistémica, eleva en forma significativa el gasto y el índice cardiaco y reduce las presiones de llenado en el tratamiento de la disfunción ventricular izquierda posoperatoria, siendo muy bien tolerado.

En cirugía cardiaca a pesar de la protección con cardioplejia se suele producir cierto grado de aturdimiento miocárdico que con frecuencia requiere soporte ino- trópico y vasopresor. El uso del levosimendan ha confirmado un claro aumento del gasto cardiaco en pacientes con contractilidad tanto normal como deprimida. ${ }^{26}$

En el síndrome de bajo gasto posoperatorio hay estudios concluyentes que confirman su mejoría con el uso de levosimendan. ${ }^{26}$ El trabajo de Levin y col. confirma que el medicamento mejora el gasto cardiaco en pacientes con bajo gasto posoperatorio. La importancia de ese estudio no deriva de este aporte, sino de los datos sobre la disminución en la morbilidad y mejoría en la supervivencia.

En nuestro estudio se documentó que los pacientes que presentaron bajo gasto cardiaco posquirúrgico y recibieron levosimendan restablecieron un adecuado índice y gasto cardiacos como lo reporta la literatura. ${ }^{26,27}$ Todos los pacientes antes de iniciar la infusión del fármaco recibían tratamientos convencionales que incluyeron el uso de diuréticos, vasodilatadores e inotrópicos. Encontramos que el índice cardiaco previo a la administración del medicamento que era de 2.7 $\mathrm{L} / \mathrm{min} / \mathrm{m}^{2}$ pasó a ser de $3.5 \mathrm{~L} / \mathrm{min} / \mathrm{m}^{2}$ y el gasto car- 
diaco de $3.5 \mathrm{~L} / \mathrm{min}$ paso a $5 \mathrm{~L} / \mathrm{min}$. De igual forma se hallaron cambios en el gasto urinario que pasó de 0.7 $\mathrm{mL} / \mathrm{k} / \mathrm{h}$ a ser de $1 \mathrm{~mL} / \mathrm{k} / \mathrm{h}$.

Hallamos también que la fracción de eyección del ventrículo izquierdo cuando se determinó deteriorada por disfunción ventricular, pasó a ser de $25 \%$ hasta $35 \%$ a la semana después de haber recibido el manejo con levosimendan, lo que nos permite inferir la respuesta benéfica de este medicamento, no reportada así en la literatura revisada en términos de fracción de eyección.

En nuestra serie de casos no se presentaron arritmias, necesidad de suspensión del tratamiento ni deterioro del comportamiento hemodinámico, como lo fue en el estudio LIDO $^{23}$, que reporta una incidencia de arritmias cardiacas de $3.9 \%$.

Reconocemos las limitaciones que presenta nuestro estudio debido a la población pequeña y al no tener un grupo control que nos impide establecer de manera categórica la asociación entre el síndrome de bajo gasto cardiaco, el uso de levosimendan en el posoperatorio y la mejoría de la función ventricular.

\section{Conclusión}

En esta serie de casos el uso de levosimendan en posoperatorio de cirugía cardiaca mejoró la fracción de eyección ventricular izquierda y además optimizó el estado hemodinámico del paciente a la semana de terminado el tratamiento.

Faltan estudios con mayor población para poder determinar el real efecto benéfico del levosimendan en este tipo de intervención.

\section{Referencias}

1. Higgins TL, Estafanous FG, Loop FD, Beck GJ, Blum JM, Paranandi L. Stratification of morbidity and mortality outcome by preoperative risk factors in coronary artery bypass patients. A clinical severity score. JAMA. 1992;267(17):23448. Epub 1992/05/06.

2. Eslava-Schmalbach JH, Rincón CJ, Guarnizo CC. "Inequidad" de la expectativa de vida al nacer por sexo y "departamentos" de Colombia. Biomédica; Vol 33 núm 3 (2013). doi: 10.7705/biomedica.v33i3.811.

3. Delgado JF. [Levosimendan in acute heart failure: past, present and future]. Rev Esp Cardiol. 2006;59(4):309-12. Epub 2006/05/20. Levosimendan en la insuficiencia cardíaca aguda: pasado, presente y futuro.
4. Breisblatt WM, Stein KL, Wolfe CJ, Follansbee WP, Capozzi J, Armitage JM, et al. Acute myocardial dysfunction and recovery: a common occurrence after coronary bypass surgery. J Am Coll Cardiol. 1990;15(6):1261-9. Epub 1990/05/01.

5. Pollesello P, Mebazaa A. ATP-dependent potassium channels as a key target for the treatment of myocardial and vascular dysfunction. Curr Opin Crit Care. 2004;10(6):436-41. Epub 2004/12/24.

6. Garlid KD, Dos Santos P, Xie ZJ, Costa AD, Paucek P. Mitochondrial potassium transport: the role of the mitochondrial ATP-sensitive $\mathrm{K}(+)$ channel in cardiac function and cardioprotection. Biochim Biophys Acta. 2003;1606(1-3):1-21. Epub 2003/09/26.

7. Malliotakis P, Xenikakis T, Linardakis M, Hassoulas J. Haemodynamic effects of levosimendan for low cardiac output after cardiac surgery: a case series. Hellenic J Cardiol. 2007;48(2):80-8. Epub 2007/05/11

8. Nashef SAM. European system for cardiac operative risk evaluation (EuroSCORE). European Journal of Cardio - Thoracic Surgery. 1999;16(1):4.

9. Mangano DT. Biventricular function after myocardial revascularization in humans: deterioration and recovery patterns during the first 24 hours. Anesthesiology. 1985;62(5):571-7. Epub 1985/05/01.

10. Tamargo J, Lopez-Sendon J. [Rationale and clinical evidence for the effects of new pharmacological treatments for heart failure]. Rev Esp Cardiol. 2004;57(5):447-64. Epub 2004/05/21. Bases y evidencias clinicas de los efectos de los nuevos tratamientos farmacologicos en la insuficiencia cardíaca.

11. Moiseyev VS, Poder P, Andrejevs N, Ruda MY, Golikov AP, Lazebnik LB, et al. Safety and efficacy of a novel calcium sensitizer, levosimendan, in patients with left ventricular failure due to an acute myocardial infarction. A randomized, placebo-controlled, double-blind study (RUSSLAN). Eur Heart J. 2002;23(18):1422-32. Epub 2002/09/05

12. Benlolo S, Lefoll C, Katchatouryan V, Payen D, Mebazaa A. Successful use of levosimendan in a patient with peripartum cardiomyopathy. Anesth Analg. 2004;98(3):822-4, table of contents. Epub 2004/02/26.

13. Cheung EH, Arcidi JM, Jr., Dorsey LM, Vinten-Johansen J, Hatcher CR, Jr., Guyton RA. Reperfusion of infarcting myocardium: benefit of surgical reperfusion in a chronic model. Ann Thorac Surg. 1989;48(3):331-8. Epub 1989/09/01.

14. Vinten-Johansen J, Chiantella V, Johnston WE, Jolly BT, Kendricks WD, Hester TO, et al. Adjuvant N-(2-mercaptopropionyl)-glycine in blood cardioplegia does not improve myocardial protection in ischemically damaged hearts. J Thorac Cardiovasc Surg. 1990;100(1):65-76. Epub 1990/07/01.

15. Yokoyama H, Julian JS, Vinten-Johansen J, Johnston WE, Smith TD, McGee DS, et al. Postischemic $[\mathrm{Ca} 2+]$ repletion improves cardiac performance without altering oxygen demands. Ann Thorac Surg. 1990;49(6):894-902. Epub 1990/06/01.

16. Cunningham JN, Jr., Adams PX, Knopp EA, Baumann FG, Snively SL, Gross RI, et al. Preservation of ATP, ultrastructure, and ventricular function after aortic cross-clamping and reperfusion. Clinical use of blood potassium cardioplegia. J Thorac Cardiovasc Surg. 1979;78(5):708-20. Epub 1979/11/01.

17. Opie LH. The heart: physiology, from cell to circulation: Lippincott-Raven; 1998 $1998.637 \mathrm{p}$

18. Kevin LG, Novalija E, Stowe DF. Reactive oxygen species as mediators of cardiac injury and protection: the relevance to anesthesia practice. Anesth Analg. 2005;101(5):1275-87. Epub 2005/10/26.

19. Kloner RA, Jennings RB. Consequences of brief ischemia: stunning, preconditioning, and their clinical implications: part 2. Circulation. 2001;104(25):3158-67. Epub 2001/12/19.

20. Follath F, Cleland JG, Just H, Papp JG, Scholz H, Peuhkurinen K, et al. Efficacy and safety of intravenous levosimendan compared with dobutamine in severe low-output heart failure (the LIDO study): a randomised double-blind trial. Lancet. 2002;360(9328):196-202. Epub 2002/07/23.

21. Luu M, Stevenson LW, Brunken RC, Drinkwater DM, Schelbert HR, Tillisch JH. Delayed recovery of revascularized myocardium after referral for cardiac transplantation. Am Heart J. 1990;119(3 Pt 1):668-70. Epub 1990/03/01.

22. Greenberg B, Borghi C, Perrone S. Pharmacotherapeutic approaches for decompensated heart failure: a role for the calcium sensitiser, levosimendan? Eur J Heart Fail. 2003;5(1):13-21. Epub 2003/02/01. 
23. Alvarez J, Bouzada M, Fernandez AL, Caruezo V, Taboada M, Rodriguez J, et al [Hemodynamic effects of levosimendan compared with dobutamine in patients with low cardiac output after cardiac surgery]. Rev Esp Cardiol. 2006;59(4):33845. Epub 2006/05/20. Comparacion de los efectos hemodinamicos del levosimendan con la dobutamina en pacientes con bajo gasto despues de cirugia cardíaca.

24. Levin RL, Degrange MA, Porcile R, Salvagio F, Blanco N, Botbol AL, et al. [The calcium sensitizer levosimendan gives superior results to dobutamine in postoperative low cardiac output syndrome]. Rev Esp Cardiol. 2008;61(5):471-9. Epub 2008/05/09. Superioridad del sensibilizante al calcio levosimendan comparado con dobutamina en el sindrome de bajo gasto cardíaco postoperatorio.
25. Garcia Gonzalez MJ, Dominguez Rodriguez A. Pharmacologic treatment of heart failure due to ventricular dysfunction by myocardial stunning: potential role of levosimendan. Am J Cardiovasc Drugs. 2006;6(2):69-75. Epub 2006/03/25.

26. Raja SG, Rayen BS. Levosimendan in cardiac surgery: current best available evidence. Ann Thorac Surg. 2006;81(4):1536-46. Epub 2006/03/28.

27. Lilleberg J, Nieminen MS, Akkila J, Heikkila L, Kuitunen A, Lehtonen L, et al. Effects of a new calcium sensitizer, levosimendan, on haemodynamics, coronary blood flow and myocardial substrate utilization early after coronary artery bypass grafting. Eur Heart J. 1998;19(4):660-8. Epub 1998/05/23. 